\title{
Predicting Positive and Negative Links in Online Social Networks
}

\author{
Jure Leskovec \\ Stanford University \\ jure@cs.stanford.edu
}

\author{
Daniel Huttenlocher \\ Cornell University \\ dph@cs.cornell.edu
}

\author{
Jon Kleinberg \\ Cornell University \\ kleinber@cs.cornell.edu
}

\begin{abstract}
We study online social networks in which relationships can be either positive (indicating relations such as friendship) or negative (indicating relations such as opposition or antagonism). Such a mix of positive and negative links arise in a variety of online settings; we study datasets from Epinions, Slashdot and Wikipedia. We find that the signs of links in the underlying social networks can be predicted with high accuracy, using models that generalize across this diverse range of sites. These models provide insight into some of the fundamental principles that drive the formation of signed links in networks, shedding light on theories of balance and status from social psychology; they also suggest social computing applications by which the attitude of one user toward another can be estimated from evidence provided by their relationships with other members of the surrounding social network.
\end{abstract}

Categories and Subject Descriptors: H.2.8 [Database Management]: Database applications-Data mining

General Terms: Algorithms; Experimentation.

Keywords: Signed Networks, Structural Balance, Status Theory, Positive Edges, Negative Edges, Trust, Distrust.

\section{INTRODUCTION}

Social interaction on the Web involves both positive and negative relationships - people form links to indicate friendship, support, or approval; but they also link to signify disapproval of others, or to express disagreement or distrust of the opinions of others. While the interplay of positive and negative relations is clearly important in many social network settings, the vast majority of online social network research has considered only positive relationships [19].

Recently a number of papers have begun to investigate negative as well as positive relationships in online contexts. For example, users on Wikipedia can vote for or against the nomination of others to adminship [3]; users on Epinions can express trust or distrust of others [8, 18]; and participants on Slashdot can declare others to be either "friends" or "foes" [2, 13, 14]. More generally, arbitrary hyperlinks on the Web can be used to indicate agreement or disagreement with the target of the link, though the lack of explicit labeling in this case makes it more difficult to reliably determine this sentiment [20].

For a given link in a social network, we will define its sign to be positive or negative depending on whether it expresses a positive or negative attitude from the generator of the link to the recipient $\mathrm{A}$

\footnotetext{
${ }^{1}$ We consider primarily the case of directed links, though our framework can be applied to undirected links as well.

Copyright is held by the International World Wide Web Conference Committee (IW3C2). Distribution of these papers is limited to classroom use, and personal use by others.

WWW 2010, April 26-30, 2010, Raleigh, North Carolina, USA. ACM 978-1-60558-799-8/10/04.
}

fundamental question is then the following: How does the sign of a given link interact with the pattern of link signs in its local vicinity, or more broadly throughout the network? Moreover, what are the plausible configurations of link signs in real social networks? Answers to these questions can help us reason about how negative relationships are used in online systems, and answers that generalize across multiple domains can help to illuminate some of the underlying principles.

Effective answers to such questions can also help inform the design of social computing applications in which we attempt to infer the (unobserved) attitude of one user toward another, using the positive and negative relations that have been observed in the vicinity of this user. Indeed, a common task in online communities is to suggest new relationships to a user, by proposing the formation of links to other users with whom one shares friends, interests, or other properties. The challenge here is that users may well have pre-existing attitudes and opinions - both positive and negative - towards others with whom they share certain characteristics, and hence before arbitrarily making such suggestions to users, it is important to be able to estimate these attitudes from existing evidence in the network. For example, if $A$ is known to dislike people that $B$ likes, this may well provide evidence about $A$ 's attitude toward $B$.

Edge Sign Prediction. With this in mind, we begin by formulating a concrete underlying task - the edge sign prediction problem - for which we can directly evaluate and compare different approaches. The edge sign prediction problem is defined as follows. Suppose we are given a social network with signs on all its edges, but the sign on the edge from node $u$ to node $v$, denoted $s(u, v)$, has been "hidden." How reliably can we infer this sign $s(u, v)$ using the information provided by the rest of the network? Note that this problem is both a concrete formulation of our basic questions about the typical patterns of link signs, and also a way of approaching our motivating application of inferring unobserved attitudes among users of social computing sites. There is an analogy here to the link prediction problem for social networks [16]; in the same way that link prediction is used to to infer latent relationships that are present but not recorded by explicit links, the sign prediction problem can be used to estimate the sentiment of individuals toward each other, given information about other sentiments in the network.

In studying the sign prediction problem, we are following an experimental framework articulated by Guha et al. in their study of trust and distrust on Epinions [8]. We extend their approach in a number of directions. First, where their goal was to evaluate propagation algorithms based on exponentiating the adjacency matrix, we approach the problem using a machine-learning framework that enables us to evaluate which of a range of structural features are most informative for the prediction task. Using this framework, we also obtain significantly improved performance on the task itself. 
Second, we investigate the problem across a range of datasets, and identify principles that generalize across all of them, suggesting certain consistencies in patterns of positive and negative relationships in online domains.

Finally, because of the structure of our learned models, we are able to compare them directly to theories of link signs from social psychology - specifically, to theories of balance and status. These will be defined precisely in Section 3, but roughly speaking, balance is a theory based on the principles that "the enemy of my friend is my enemy," "the friend of my enemy is my enemy," and variations on these [4, 11]. Status is a theory of signed link formation based on an implicit ordering of the nodes, in which a positive $(u, v)$ link indicates that $u$ considers $v$ to have higher status, while a negative $(u, v)$ link indicates that $u$ considers $v$ to have lower status. The point is that each of these theories implicitly posits its own model for sign prediction, which can therefore be compared to our learned models. The result is both a novel evaluation of these theories on large-scale online data, and an illumination of our learned models in terms of where they are consistent or inconsistent with these theories.

Generalization across Datasets. We study the problem of sign prediction on three datasets from popular online social media sites; in all cases, we have network data with explicit link signs. The first is the trust network of Epinions, in which the sign of the link $(u, v)$ indicates whether $u$ has expressed trust or distrust of user $v$ (and by extension, the reviews of $v$ ) [8]. The second is the social network of the technology blog Slashdot, where $u$ can designate $v$ as either a "friend" or "foe" to indicate $u$ 's approval or disapproval of $v$ 's comments [2] 13 14]. The third is the voting network of Wikipedia; here, the sign of the link $(u, v)$ indicates whether $u$ voted for or against the promotion of $v$ to admin status [3].

Despite the fact that link signs have quite different meanings in the three settings, our main results generalize across all three domains in several important ways. First, we find that sign prediction performance degrades only slightly when we train our models on one domain and test them on another. This indicates that our models are capturing principles that will arguably generalize to a range of future contexts in which signed links are employed, rather than picking up on idiosyncrasies of particular individual domains. Moreover, this generalization holds despite the fact that the quality of prediction performance is different across the domains: for example, predicting link signs is more difficult on Wikipedia, yet models trained on Wikipedia still perform on other domains with very little loss of accuracy compared to models that were explicitly trained on those domains.

Second, we find that the social-psychological theories of balance and status agree with the learned models in certain characteristic ways, and disagree in other characteristic ways, as we elaborate in Section 3 These similarities and contrasts among the models likewise persist at a general level across the datasets, and thus provide insight into the successes and failures of balance and status as interpretative frameworks for understanding how link signs are being used across all these systems.

Additional Tasks. We consider several further issues beyond the problem of sign prediction. Among these, we ask whether information about negative links can be helpful in addressing questions that concern purely positive links. Specifically we consider the link prediction problem: given a pair $u$ and $v$, is there a (hidden) positive edge between $u$ and $v$ ? We ask how much performance is improved if the negative edges in the network are also visible. In other words, how useful is it to know where a person's enemies are, if we want to predict the presence of additional friends? We find that negative links can be a powerful source of additional informa- tion for a task such as this: on all two of the three datasets, we get a boost in improvement over random choice of up to a factor of 1.5. This type of result helps to argue that positive and negative links in online systems should be viewed as tightly related to each other, rather than as distinct non-interacting features of the system.

We also investigate more "global" properties of signed social networks, motivated by the local theories of balance and status. Specifically, the "friend of my enemy" logic of balance theory suggests that if balance is a key factor in determining signed link formation at a global scale, then we should see the network partition into large opposed factions. The logic of status theory, on the other hand, suggests that we should see an approximate total ordering of the nodes, with positive links pointing from left to right and negative links pointing from right to left. Searching for either of these global patterns involves developing approximate optimization heuristics for the underlying networks, since the two patterns correspond roughly to the well-known maximum cut and maximum acyclic subgraph problems. We employ such heuristics, and find significant evidence for the global total ordering suggested by status theory, but essentially no evidence for the division into factions suggested by balance theory. This result provides an intriguing contrast with our basic results on sign prediction using local features, where strong aspects of both theories are present; it suggests that the mechanisms by which local organizing principles scale up to global ones is complex, and an interesting source of further open questions.

Further Related Work. Earlier in the introduction, we discussed some of the main lines of research on which we are building; here, we survey further lines of study that are also related to our work.

First, our use of trust networks as a source of data connects to a large body of work on trust management in several settings, including peer-to-peer networks [12] 25], Semantic Web applications [22], and Web spam detection [10]. Related to trust management is the development of user rating mechanisms on sites such as Slashdot [13, 14] and the development of norms to control deviant behavior [6]. Recent work has also investigated online communities devoted to discussion of controversial topics, where one can expect to find strong positive and negative interactions [2, 24]; and the analysis of sentiment, subjectivity, and opinion in text has become an active area in natural language processing [20].

Our general goal of inferring an individual's attitudes suggests parallels to a long line of work on recommendation systems [21], in which the goal is typically to infer how a user would evaluate given items based on their evaluation of other items. There are crucial differences, however, between an analysis in which a user is evaluating (inert) items, and our case in which a user is evaluating other people — in this latter case, the objects being evaluated are themselves capable of forming opinions and expressing attitudes, and this provides additional sources of information based on the full social network of interactions.

As noted above, there is a long history of work in the social sciences on balance theory [4, 11], including more recent work on mathematical models that attempt to capture how balance can arise from dynamic changes to link signs over time [1, 17]. In recent work, we analyzed theories of balance and status in the context of social media sites, investigating the extent to which each theory helped explain the linking behavior of users on these sites [15]. Our work there studied how balance and status effects can act as modifiers on the default behavior of a set of people measured in aggregate; the problem of making predictions at the level of individuals was left as an open question. Here, we take some initial steps toward addressing this question, combining an analysis of signed networks with machine-learning techniques so as to formulate individual-level predictions. 


\begin{tabular}{l||r|r|r} 
& Epinions & Slashdot & Wikipedia \\
\hline \hline Nodes & 119,217 & 82,144 & 7,118 \\
Edges & 841,200 & 549,202 & 103,747 \\
+ edges & $85.0 \%$ & $77.4 \%$ & $78.7 \%$ \\
- edges & $15.0 \%$ & $22.6 \%$ & $21.2 \%$
\end{tabular}

Table 1: Dataset statistics.

\section{DATASET DESCRIPTION}

We consider three large online social networks where each link is explicitly labeled as positive or negative: Epinions, Slashdot and Wikipedid2.

Epinions is a product review Web site with a very active user community. Users are connected into a network of trust and distrust, which is then combined with review ratings to determine which reviews are most authoritative. The data spans from the inception of the site in 1999 until August 12, 2003. The network contains 119,217 nodes and 841,000 edges, of which $85.0 \%$ are positive. 80,668 users received at least one trust or distrust edge, while there are 49,534 users that created at least one and received at least one signed edge.

Slashdot is a technology-related news website. In 2002 Slashdot introduced the Slashdot Zoo which allows users to tag each other as "friends" or "foes." The semantics of a signed link is similar to Epinions, as a friend relation means that a user likes another user's comments, while a foe relationship means that a user finds another user's comments uninteresting. We crawled Slashdot in February 2009 to obtain its network of 82,144 users and 549,202 edges of which $77.4 \%$ are positive. 70,284 users received at least one signed edge, and there are 32,188 users with non-zero in- and out-degree.

Wikipedia is a collectively authored encyclopedia with an active user community. The network we study corresponds to votes cast by Wikipedia users in elections for promoting individuals to the role of admin. A signed link indicates a positive or negative vote by one user on the promotion of another ( + for a supporting vote and - for an opposing vote). Using the latest complete dump of Wikipedia page edit history (from January 2008) we extracted all administrator election and vote history data. This gave us 2,794 elections with 103,747 total votes and 7,118 users participating in the elections (either casting a vote or being voted on). Out of this total, 1,235 elections resulted in a successful promotion, while 1,559 elections did not result in the promotion of the candidate. About half of the votes in the dataset are by the existing admins, while the other half comes from ordinary Wikipedia users. The resulting network contains 7,118 nodes and 103,747 edges of which $78.7 \%$ are positive. There are 2,794 nodes that receive at least one edge and 1,376 users that both received and created signed edges.

In all networks the background proportion of positive edges is about the same, with $\approx 80 \%$ of the edges having a positive sign.

\section{PREDICTING EDGE SIGN}

We now consider the problem of predicting the sign of individual edges in our dataset. The set-up for this problem follows the framework of Guha et al. [8]: We are given a full network with all but one of the edge signs visible, and we are interested in predicting the sign of this single edge whose sign has been suppressed. This can be viewed as leave-one-out cross-validation in the present context, where we learn using the rest of the network and aim to predict the missing sign of a single edge.

\subsection{A Machine-Learning Formulation}

Given a directed graph $G=(V, E)$ with a sign (positive or negative) on each edge, we let $s(x, y)$ denote the sign of the edge $(x, y)$

\footnotetext{
${ }^{2}$ Datasets are available at http: / / snap. stanford.edu
}

from $x$ to $y$. That is, $s(x, y)=1$ when the sign of $(x, y)$ is positive, -1 when the sign is negative, and 0 when there is no directed edge from $x$ to $y$. Sometimes we will also be interested in the sign of a directed edge connecting $x$ and $y$, regardless of its direction; thus, we write $\bar{s}(x, y)=1$ when there is a positive edge in one of the two directions $(x, y)$ or $(y, x)$, and either a positive edge or no edge in the other direction. We write $\bar{s}(x, y)=-1$ analogously when there is a negative edge in one of these directions, and either a negative edge or no edge in the other direction. We write $\bar{s}(x, y)=0$ in all other cases (including when there are edges $(x, y)$ and $(y, x)$ with opposite signs, though this is in fact rare in our datasets). For different formulations of our task, we will suppose that for a particular edge $(u, v)$, the sign $s(u, v)$ or $\bar{s}(u, v)$ is hidden and that we are trying to infer it.

Features. We begin by defining a collection of features for our initial machine-learning approach to this problem. The features are divided into two classes. The first class is based on the (signed) degrees of the nodes, which essentially record the aggregate local relations of a node to the rest of the world. The second class is based on the principle from social psychology that we can understand the relationship between individuals $u$ and $v$ through their joint relationships with third parties $w$ : for example, is there someone who has a positive relationship toward both $u$ and $v$, a negative relationship toward both $u$ and $v$, or a positive relationship toward one and a negative relationship toward the other? Thus, features of this second class are based on two-step paths involving $u$ and $v$.

We define the first class of features, based on degree, as follows. As we are interested in predicting the sign of the edge from $u$ to $v$, we consider outgoing edges from $u$ and incoming edges to $v$. Specifically we use $d_{i n}^{+}(v)$ and $d_{i n}^{-}(v)$ to denote the number of incoming positive and negative edges to $v$, respectively. Similarly we use $d_{\text {out }}^{+}(u)$ and $d_{\text {out }}^{-}(u)$ to denote the number of outgoing positive and negative edges from $u$, respectively. We use $C(u, v)$ to denote the total number of common neighbors of $u$ and $v$ in an undirected sense - that is, the number of nodes $w$ such that $w$ is linked by an edge in either direction with both $u$ and $v$. We will also refer to this quantity $C(u, v)$ as the embeddedness of the edge $(u, v)$. Our seven degree features are the five quantities $d_{i n}^{+}(u), d_{i n}^{-}(v), d_{\text {out }}^{+}, d_{\text {out }}^{-}$, and $C(u, v)$, together with the total out-degree of $u$ and the total in-degree of $v$, which are $d_{\text {out }}^{+}(u)+d_{\text {out }}^{-}(u)$ and $d_{\text {in }}^{+}(v)+d_{\text {in }}^{-}(v)$ respectively.

For the second class of feature we consider each triad involving the edge $(u, v)$, consisting of a node $w$ such that $w$ has an edge either to or from $u$ and also an edge either to or from $v$. There are 16 distinct types of triads involving $(u, v)$ : the edge between $w$ and $u$ can be in either direction and of either sign, and the edge between $w$ and $v$ can also be in either direction and of either sign; this leads to $2 \cdot 2 \cdot 2 \cdot 2=16$ possibilities. Each of these 16 triad types may provide different evidence about the sign of the edge from $u$ to $v$, some favoring a negative sign and some favoring a positive sign. We encode this information in a 16-dimensional vector specifying the number of triads of each type that $(u, v)$ is involved in.

Learning Methodology and Results. We use a logistic regression classifier to combine the evidence from these individual features into an edge sign prediction. Logistic regression learns a model of the form

$$
P(+\mid x)=\frac{1}{\left.1+e^{-\left(b_{0}+\sum_{i}^{n} b_{i} x_{i}\right.}\right)}
$$

where $x$ is a vector of features $\left(x_{1}, \ldots, x_{n}\right)$ and $b_{0}, \ldots, b_{n}$ are the coefficients we estimate based on the training data.

The edges signs in the networks that we study are overwhelmingly positive. Thus we consider and evaluate two different approaches. First, we use the full dataset where about $80 \%$ of the 

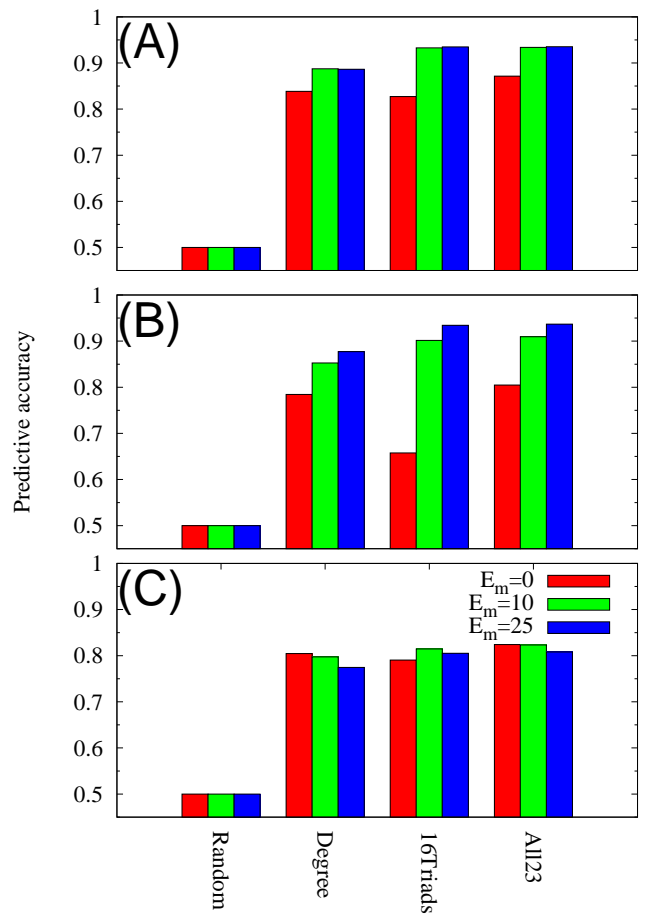

Figure 1: Accuracy of predicting a sign of edge $(u, v)$ given signs of all other edges in the network. (a) Epinions, (b) Slashdot, (c) Wikipedia.

edges are positive. Second, we follow the methodology of Guha et al. [8] and create a balanced dataset with equal numbers of positive and negative edges, so that random guessing yields a 50\% correct prediction rate. For every negative edge $(u, v)$ we sample a random positive edge, which ensures that the number of positive and negative edges in the data we consider for training and prediction is balanced. Moreover, we also consider two different evaluation measures: the classification accuracy and the area under the ROC curve (AUC). For ease of exposition we focus on classification accuracy on a balanced dataset. As we discuss later our results are robust to whether we use the full or balanced dataset and whether we evaluate using AUC or accuracy.

We describe each edge $(u, v)$ in this set using the two classes of features described above. We consider all 23 features together, and we also evaluate performance using features of each class separately — that is, representing each edge as a 7-dimensional vector of degree features and as a 16-dimensional vector of triad features. We also consider performance across different types of edges. In particular, since the triad features are relevant only when $u$ and $v$ have neighbors in common, it is natural to expect that they will be most effective for edges of greater embeddedness. We therefore consider the performance restricted to subsets of edges of different levels of minimum embeddedness.

The classification accuracy is shown in Figure 1, where results are described for all three datasets, for the two classes of features separately and together, and for different levels of minimum embeddedness (denoted by $E_{m}$ ). Several observations stand out. First, prediction based on the learned models significantly outperform the results reported in Guha et al. [8] for the Epinions dataset. The lowest error rate achieved in their paper is $14.7 \%$ whereas we obtain error rates of $11.45 \%$ for Degree, $6.64 \%$ for 16 Triads and $6.58 \%$ for All23.

These results are particularly interesting because our features are based only on local properties in the one-step neighborhood of the edge $(u, v)$ whose sign is being inferred, in contrast with the prop- agation model of Guha et al. This suggests that edge signs can be meaningfully understood in terms of such local properties, rather than requiring a notion of propagation from farther-off parts of the network.

Second, consistent with intuition, the triad features perform less well than the degree features for edges of low embeddedness, but become more effective as the embeddedness increases and a greater amount of triadic information becomes available.

Finally, it is also noteworthy that the accuracy on the Wikipedia network is significantly lower than on the other two networks, even for edges with large embeddedness. This discrepancy between Wikipedia and the other datasets is interesting because the positive and negative links on Wikipedia correspond to evaluations that are more publicly visible, more consequential, and more informationbased than for the other two datasets, since they result from public votes on promotion of individuals to adminship, where the candidates being voted on have accumulated a long history of activity on Wikipedia. One could conjecture that these aspects of the evaluations in the Wikipedia dataset make it correspondingly more difficult (though still surprisingly feasible) to predict their outcomes from simple structural measures.

In all experiments we report the average accuracy and estimated logistic regression coefficients over 10 -fold cross validation. If not stated otherwise, we limit our analyses to edges with minimum embeddedness 25 . We note that our results are robust with respect to training dataset and evaluation metric. Generally, when using the full dataset rather than the balanced one, random guessing improves accuracy from $50 \%$ to approximately $80 \%$. With the full dataset the accuracy of our logistic regression method correspondingly jumps to the $90-95 \%$ range and maintains roughly a $15 \%$ absolute improvement over random guessing. When evaluating using AUC rather than accuracy the overall pattern of performance does not change. The various forms of logistic regression have AUC of approximately $90 \%$ on the balanced dataset and $95 \%$ on the full dataset.

\subsection{Connections to Theories of Balance and Status}

Our goal is to use the machine learning framework not just to predict the edge signs themselves, but also for deriving insights into the usage of these systems based on the observed patterns of positive and negative edges.

Specifically, logistic regression provides a coefficient associated with each feature, which suggests how the feature is being used by the model to provide weight for or against a positive edge sign. This provides a natural and appealing connection to classical theories from social psychology, which also offer proposals for how subsets of these features offer evidence for the sign of the edge $(u, v)$.

We focus here on the second class of features, based on triad types, which are motivated by social-psychological theories about local patterns of relationships. Specifically, we say that a theory of triad types is a function

$$
f:\{\text { types } \tau\} \rightarrow\{+1,-1,0\},
$$

which specifies for each triad type $\tau$ whether it constitutes evidence for a positive $(u, v)$ edge $(f(\tau)=+1)$, evidence for a negative $(u, v)$ edge $(f(\tau)=-1)$, or whether it offers no evidence $(f(\tau)=$ $0)$.

Our logistic regression model provides a learned theory of triad types for each dataset, in which $f(\tau)$ is equal to the sign of the coefficient associated with the feature $\tau$. But several principles from social psychology also provide theories of triad types, developed from plausible assumptions about human behavior rather than through a data-driven approach. In other words, the learned model 
and the qualitative models from the literature are expressed in the same language - as mappings from triad types to positive or negative evidence - and we can thus ask questions about how the theories align with each other. Through this line of investigation we can gain insight into two issues: first, we can evaluate the existing theories on our on-line datasets; and second, we can use these existing theories as an interpretive framework for reasoning about the structure of our learned model.

Balance and Status. We begin by summarizing the two main social-psychological theories of triad types that we compare to.

The more well-studied of the two is structural balance theory, based on the common principles that "the friend of my friend is my friend," "the enemy of my friend is my enemy," "the friend of my enemy is my enemy," and (perhaps less convincingly) "the enemy of my enemy is my friend." Concretely, this means that if $w$ forms a triad with the edge $(u, v)$, then structural balance theory posits that $(u, v)$ should have the sign that causes the triangle on $u, v, w$ to have an odd number of positive signs, regardless of edge direction - just as each of the principles above has an odd number of occurrences of the word "friend." In other words, $f_{\text {balance }}(\tau)=$ $\bar{s}(u, w) \bar{s}(v, w)$, where we recall that the value of $\bar{s}$ corresponds to the sign regardless of the direction of the edge.

An alternate theory, which is implicit in the work of Guha et al. [8] and developed further in our recent research [15], is a theory of status. In this theory, a positive edge $(x, y)$ means that $x$ regards $y$ as having higher status than herself, while a negative edge $(x, y)$ means that $x$ regards $y$ as having lower status than herself. Assuming that all participants in the system agree on this status ordering, status theory predicts that when the direction of an edge is flipped, its sign should flip as well.

So to determine $f_{\text {status }}(\tau)$, we first flip the directions of the edges between $u$ and $w$ and between $v$ and $w$, if necessary, so that they point from $u$ to $w$ and from $w$ to $v$; we flip the signs accordingly as we do this. We then define $f_{\text {status }}(\tau)$ to be the sign of $s(u, w)+s(w, v)$. This means that status theory makes no prediction when the two signs cancel out, but otherwise, it predicts a positive or negative sign based on the imputed status relationship between $u$ and $v$.

Notice that balance and status agree on some types of triads for example, when $u$ points positively to $w$ and $w$ points positively to $v$, then $v$ is both the friend of $u$ 's friend, and also someone of higher status than $u$, and thus both theories predict a positive sign for $(u, v)$. But balance and status can also disagree - for example, when $v$ points positively to $w$ and $w$ points positively to $u$, then balance concludes that $v$ is the friend of $u$ 's friend and thus $(u, v)$ is positive, but status posits that $v$ has lower status than $u$ and thus $(u, v)$ is negative.

Comparison of Balance and Status with the Learned Model. In Table 2. we show the signs of the three theories discussed above - balance, status, and the learned model — on the three datasets. For denoting the 16 triad types, in the table and elsewhere, we use a shorthand in which we record the four binary choices that comprise each type. Thus a type $\tau$ will be represented by a string of the form $[F \mid B][F \mid B][p \mid m][p \mid m]$ to indicate the direction of the edges along the two-step path $u-w-v$ (Forward or Backward on each step), and the signs of these two edges (plus or minus). For example, FBmp is the triad type in which $u$ points negatively to $w$, and $v$ points positively to $w$ (since the first step in the $u-w-v$ path is forward and minus, while the second is backward and plus).

At a general level, the results show that both social-psychological theories agree fairly well with the learned models - with agreement on more than half the triad types where they make predictions, and generally on three-quarters or more of the triad types. Look-

\begin{tabular}{l||r|r|r|r} 
Feature & Balance theory & Epinions & Slashdot & Wikipedia \\
\hline \hline const & 0 & 0.4321 & 1.4973 & 0.0395 \\
pp & 1 & 0.0470 & 0.0395 & 0.0553 \\
pm & -1 & -0.1154 & -0.2464 & -0.1632 \\
mp & -1 & -0.2125 & -0.3476 & -0.1432 \\
mm & 1 & -0.0149 & -0.0262 & -0.0465
\end{tabular}

Table 3: Regression coefficients based on Balance attributes and learned logistic regression.

\begin{tabular}{l||r|r|r|r} 
Feature & Status theory & Epinions & Slashdot & Wikipedia \\
\hline \hline const & 0 & -0.6873 & -1.3915 & -0.3039 \\
$u<w<v$ & 1 & 0.1165 & 0.0463 & 0.0258 \\
$u>w>v$ & -1 & -0.1002 & -0.114 & -0.1941 \\
$u<w>v$ & 0 & 0.0572 & 0.1558 & 0.0300 \\
$u>w<v$ & 0 & -0.0064 & 0.0382 & 0.0543
\end{tabular}

Table 4: The coefficients based on Status Theory and learned logistic regression.

ing at the absolute values of the coefficients, we note that certain features stand out in importance across all three datasets - specifically, there are coefficients of large magnitude for all the FF features, as well as the FBmp and BFpm features. In contrast, the BB features have coefficients of much smaller magnitude. We also see that balance theory is in notably better alignment with the learned model for Epinions and Slashdot than it is for Wikipedia. As discussed above, Wikipedia differs considerably from the other two datasets in that it is a publicly visible voting forum. Given these results it is interesting to conjecture that in such a setting status may play a stronger role.

It is also interesting to consider the cases in which there are relatively stable disagreements among the models across the three datasets. In particular, we see that balance theory consistently disagrees with the learned model (and with status theory) when it predicts that a negative $(u, w)$ and negative $(w, v)$ edge should suggest a positive $(u, v)$ edge. This is precisely the kind of case that seems somewhat suspect intuitively, namely "the enemy of my enemy is my friend". Balance theory also consistently disagrees with both the learned model and status theory when it predicts that a positive $(v, w)$ and positive $(w, u)$ edge should result in a positive $(u, v)$ edge. Here the direction of the two-step path is from $v$ to $u$ rather than $u$ to $v$, and one can conjecture that this opposite direction path has a lower predictive power for the $(u, v)$ sign. Indeed these two cases suggest modifications of the models, as we now discuss.

Comparison of Balance and Status with Reduced Models. To fully understand the relationship of the learned model to the theories of balance and status, it helps to look at "reductions" of the model that capture just the features essential to these two theories.

Let's begin by considering balance theory. Balance theory has generally been applied as a theory of undirected graphs, although its extension to directed graphs by ignoring edge direction (as we use it here) is standard as well [23]. With this in mind, let's consider the learning problem using a feature set in which we treat all edges as undirected. In this setting, there are only four different triad types involving a node $w$ and the edge $(u, v)$, depending on whether the undirected edge $\{u, w\}$ is positive or negative, and whether the undirected edge $\{w, v\}$ is positive or negative (since we can no longer observe the directions of these edges). Thus, we create a 4-dimensional feature vector for the edge $(u, v)$, by simply counting how many undirected triads of each type it is involved in. We then apply logistic regression to this 4-dimensional problem.

The results are depicted in Table 3 . We see that for all triad types other than the "enemy of my enemy" type $(\mathrm{mm})$, and all three datasets, the learned coefficient is the same as the prediction of balance theory. The disagreement for the $m m$ type is a further 


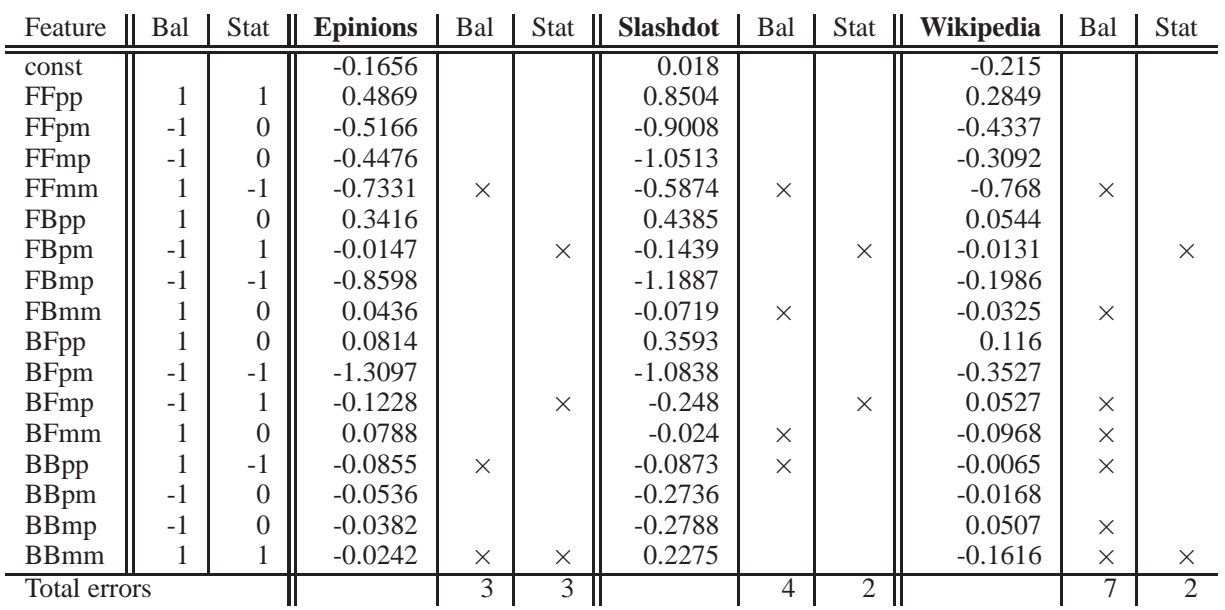

Table 2: Logistic regression coefficients compared to status and structural balance theory. $\times$ means there is discrepancy in predictions between the Balance (Status) theory and what is learned from the logistic regression model. Each line represents directions and signs of the edges on a path $(A, B, C)$ where "BFpm" stands for Backward Forward plus minus and denotes a path $A \leftarrow+B \rightarrow-C$.

\begin{tabular}{l||r|r|r} 
Feature & Epinions & Slashdot & Wikipedia \\
\hline \hline const & -2.2152 & -2.8475 & -1.4496 \\
FF & 0.2023 & 0.2092 & 0.0773 \\
FB & 0.1286 & 0.1698 & 0.0286 \\
BF & 0.0077 & 0.0842 & 0.0544 \\
BB & -0.0692 & -0.0293 & -0.0259
\end{tabular}

Table 5: Learned logistic regression coefficients for the model based on the counts of directed positive paths.

indication of the difficulty of the "enemy of my enemy" aspect of balance in these domains, and in fact is consistent with an alternative formulation of balance theory due to Davis in the 1960s [7], which agrees with standard balance theory on the first three triad types and makes no prediction on $\mathrm{mm}$. We will refer to Davis's variant on balance theory as weak balance.

We can do a similar reduction for status theory. We begin by preprocessing the graph to flip the direction and sign of each negative edge, thereby creating a positive edge with the same interpretation under status theory. The resulting graph has only positive edges, and hence there are only four triad types - based on whether the $(u, w)$ edge is forward or backward, and whether the $(w, v)$ edge is forward or backward. We create a 4-dimensional feature vector for the edge $(u, v)$ by counting the frequencies of these four triad types, and apply logistic regression.

The learned coefficients for this problem are shown in Table 4 Here we see that on both triad types for which status theory makes a prediction, and across all three datasets, the sign of the learned coefficient is the same as the sign of the status prediction.

What emerges from the analysis of these reduced-form models is that each of balance theory and status theory are essentially accurate at the granularity in which they are most naturally formulated - in the case of balance, on undirected graphs; and in the case of status, once edge signs and directions have been canonicalized. This makes it clear that our results for the more detailed 16-type model go beyond the scale of resolution at which either balance or status can provide accurate predictions, and illuminate some more subtle effects that govern social interactions.

To further elaborate on this point, we can evaluate the prediction accuracy of each of these reduced models in comparison to the full model on all 16 triad types. The results are shown in Figure 2. Here, we consider the two kinds of reduced 4-dimensional feature sets, and evaluate performance using both the coefficients learned via logistic regression (denoted BalanceLrn and StatusLrn in the figure), as well as the lower performance using coefficients from
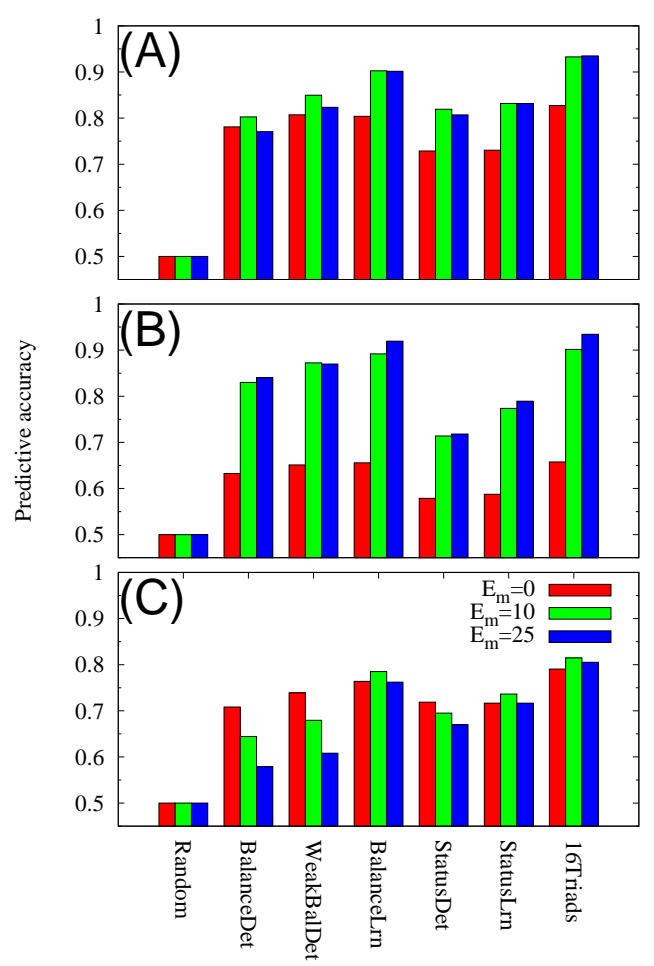

Figure 2: Accuracy of predicting a sign of edge $(u, v)$ given signs of all other edges in the network. (a) Epinions, (b) Slashdot, (c) Wikipedia.

$\{-1,0,+1\}$ provided by balance, Davis's notion of weak balance, and status (denoted BalanceDet, WeakBalDet, and StatusDet in the figure).

All-positive subgraphs. There is a final reduced model that also provides insight into the role of balance theory particularly for these datasets. Suppose that we preprocess the graph by simply deleting all negative edges, so that we are left with the subgraph consisting of only positive edges. Again, there are now four possible triad types, and we show the learned coefficients for logistic regression on this 4-dimensional problem in Table 5 Balance would predict that all coefficients should be positive, since all relations indicate friendship under the interpretation of balance theory. This agrees 


\begin{tabular}{l||r|r|r} 
All23 & Epinions & Slashdot & Wikipedia \\
\hline \hline Epinions & 0.9342 & 0.9289 & 0.7722 \\
Slashdot & 0.9249 & 0.9351 & 0.7717 \\
Wikipedia & 0.9272 & 0.9260 & 0.8021
\end{tabular}

Table 6: Predictive accuracy when training on the "row" dataset and evaluating the prediction on the "column" dataset.

with Table 5 except for the last row, where the coefficient of the learned model is negative across all three datasets. This corresponds to the triad type in which $v$ links positively to $w$, and $w$ links positively to $u$. The data indicates that this in fact serves as evidence of a negative $(u, v)$ link in all datasets, and status theory provides one simple hypothesis for why: if $v$ regards $w$ as having higher status, and $w$ in turn regards $u$ as having higher status, then arguably $u$ will view $v$ as having lower status.

\subsection{Generalization across datasets}

We now turn to the question of how well the learned predictors generalize across the three datasets, in order to investigate the extent to which the learned models are based on specific properties of each dataset versus more general properties of social network data. That is, in our investigation thus far the learned models have been able to take advantage of properties of each specific dataset, whereas the base social science models are generic across datasets. Thus it could be the case that the models obtained using logistic regression perform well only on the individual datasets on which they were trained. On the other hand, if the learned models are able to generalize across datasets it suggests that there are underlying general principles that guide the creation of signed edges in social network graphs and which the regression models are able to capture.

To evaluate the generalization accuracy of the models we perform the following experiment. For each pair of datasets we train the logistic regression model on the first dataset and evaluate it on the second dataset. Table 6 shows the results of these 9 experiments using the All23 model. The diagonal entries of the table show the results already presented above (i.e. leave-one-out crossvalidation for a single dataset), whereas the off-diagonal entries show the generalization across datasets. We see the same overall pattern as before, with prediction accuracy being considerably lower for Wikipedia than for the other two dataset. We also see that the off-diagonal entries are nearly as high as the diagonals, showing that there is very good generalization and thus there indeed are general cross-dataset properties captured by the model. In both the first and second columns (testing on the Epinions and Slashdot datasets) there is remarkably little decrease in performance regardless of which of the three datasets is used for training. Note that in particular, even training on the Wikipedia dataset yields good prediction performance on the other two datasets - thus while the Wikipedia dataset is difficult in terms of prediction accuracy it seems to provide the same underlying structural information as the other datasets in that it allows training of a more general model.

Table 7 shows the results of these experiments using the learned Balance and Status models considered above. For comparison we also show the performance of the basic BalanceDet, WeakBalance and StatusDet models (which are not learned from the data). For balance we see that the generalization performance is again very good, and also that the prediction accuracy in all cases is higher than for the nonlearned balance models. However for status, we see a considerably different picture. The model does not generalize as well, and in fact often performs worse than the baseline status model when trained on one dataset and tested on another. This suggests that the learned balance properties are relatively generic across different online settings, whereas the status properties are more particular to a given dataset.

\begin{tabular}{l||r|r|r} 
BalanceLrn & Epinions & Slashdot & Wikipedia \\
\hline \hline Epinions & 0.9027 & 0.9166 & 0.7319 \\
Slashdot & 0.9020 & 0.9203 & 0.7439 \\
Wikipedia & 0.8985 & 0.9145 & 0.7558 \\
\hline BalanceDet & 0.7709 & 0.8414 & 0.5778 \\
WeakBalance & 0.8233 & 0.8698 & 0.6081
\end{tabular}

\begin{tabular}{l||r|r|r} 
StatusLrn & Epinions & Slashdot & Wikipedia \\
\hline \hline Epinions & 0.8313 & 0.7514 & 0.6410 \\
Slashdot & 0.7682 & 0.7847 & 0.6094 \\
Wikipedia & 0.7592 & 0.6598 & 0.7163 \\
\hline StatusDet & 0.8083 & 0.7173 & 0.6679
\end{tabular}

Table 7: Predictive accuracy when training on the "row" dataset and evaluating the prediction on the "column" dataset.

\subsection{Heuristic Predictors}

Having now explored the relationship of balance and status to the output of a machine learning model, it is natural to also investigate simple "hand-written" heuristic predictors to determine baseline performance levels for the sign prediction task.

We construct a few such predictors using ideas drawn from status and balance theory, as well as considerations of node degree. We again use the same evaluation framework, in which for every negative edge $(u, v)$ we sample a random positive edge, to ensure that random guessing gets $50 \%$ of the predictions correct.

We consider the following heuristic predictors:

- A balance heuristic. For each choice of the sign of $(u, v)$, some of the triads it participates in will be consistent with balance theory, and the rest of the triads will not. We choose the sign for $(u, v)$ that causes it to participate in a greater number of triads that are consistent with balance.

- A status heuristic. We define an estimate of a node $x$ 's status to be $\sigma(x)=d_{\text {in }}^{+}(x)+d_{\text {out }}^{-}(x)-d_{\text {out }}^{+}(x)-d_{\text {in }}^{-}(x)$. This gives $x$ status benefits for each positive link it receives and each negative link it generates, and status detriments for each negative link it receives and each positive link it generates. We then predict a positive sign for $(u, v)$ if $\sigma(u) \leq \sigma(v)$, and a negative sign otherwise.

- An out-degree heuristic. We predict the majority sign based on the signs given by the edge initiator $u$. That is, we predict + if $d_{\text {out }}^{+}(u) \geq d_{\text {out }}^{-}(u)$.

- An in-degree heuristic. We predict the majority sign based on the signs received by the edge target $v$. That is, we predict + if $d_{i n}^{+}(v) \geq d_{i n}^{-}(v)$.

We show the results of these simple predictors in Figure 3 plotted as a function of embeddedness. First, while these predictors perform considerably better than guessing - and quite well in some cases - they generally perform worse than the models discussed above. Second, in the case of the Epinions data, some of these simple predictors perform comparably well to the results reported in [8] (though not as well as the very best results in that paper). This underscores the value of having simple baseline measures.

There are several additional points worth noting. First, the indegree heuristic (InSign) performs relatively poorly across all datasets (although it beats the other predictors for low embeddeness in the Wikipedia dataset), while out-degree (OutSign) performs well on two of the three; this corresponds to the intuitively natural conclusion that the generator of the edge plays a larger role in sign determination than the recipient. Second, the triadic features - status (StatDif) and balance (Balance) — do better with increasing embeddedness, but in most cases performance of these features starts to drop again as embeddedness gets too high. One can understand this in terms of a tradeoff between two types of information sparsity. On the one hand, when an edge participates in relatively few 


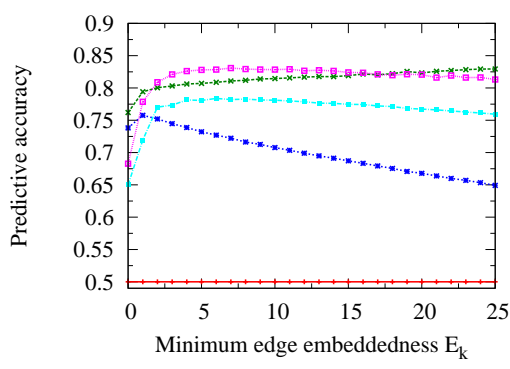

(a) Epinions

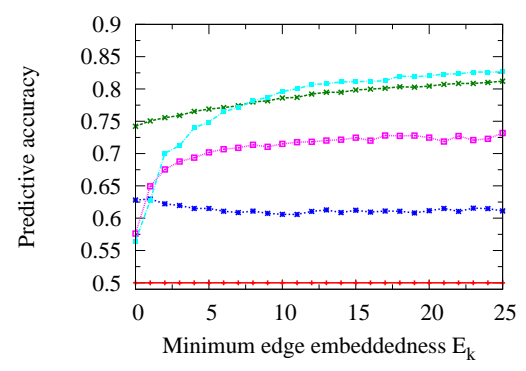

(b) Slashdot

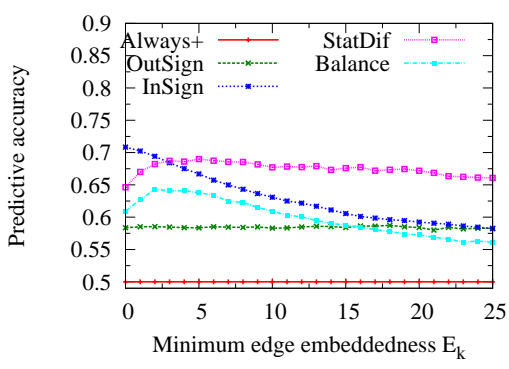

(c) Wikipedia

Figure 3: Accuracy for simple models as a function of minimum edge embeddedness. Refer to main text for model descriptions.

triads (low embedding) then the triadic features provide relatively little information, but on the other hand relatively few edges in the graph have a high degree of embedding (e.g., significantly above 25).

\section{GLOBAL STRUCTURE OF SIGNED NET- WORKS}

When we perform sign prediction for an edge $(u, v)$ using information about the two-step paths from $u$ to $v$, and when we relate our learned models to the predictions of balance and status, we are using local information about the neighborhoods of the nodes. However, the theories of balance and status also each make global predictions about the pattern of the signs in the network as a whole, and it is interesting to investigate the extent to which these global predictions are borne out by the network structure of our datasets.

Balance and Status: From Local to Global. The global predictions of balance and status are best explained in their simplest settings, which are for networks in which each pair of nodes is connected by an edge. This complete connectivity clearly does not hold for our datasets, but we will explain how to adapt the predictions of the models to our setting nonetheless, and then search for evidence of these predictions.

The global prediction of balance theory is contained in a theorem of Cartwright and Harary from the 1950s. It asserts that if all triangles in a completely connected undirected graph obey structural balance, then globally the network can be divided into two cliques of mutual friends, such that all edges between the two cliques are negative [4].

THEOREM 1 (CARTWRIGHT-HARARY). Let $G$ be a signed, undirected complete graph in which each triangle has an odd number of positive edges. Then the nodes of $G$ can be partitioned into two sets $A$ and $B$ (where one of $A$ or $B$ may be empty), such that all edges within $A$ and $B$ are positive, and all edges with one end in $A$ and the other in $B$ are negative.

We can analogously formulate a local-to-global connection for status theory, but it leads to a quite different structural prediction. Rather than undirected complete graphs, the basic form of this theorem will use completely connected directed graphs, which are referred to as tournaments: directed graphs in which each pair of nodes is connected by a directed edge in one direction or the other.

First, if we consider the local condition that motivated status theory in the previous section, it required that for any edge $(u, v)$, and any third node $w$, it should be possible to assign distinct numerical "status values" to $u, v$, and $w$ in such a way that the positive edges among them (if any) go from nodes of lower status to nodes of higher status, and the negative edges among them (if any) go from nodes of higher status to nodes of lower status. Let us say that the three nodes $u, v$, and $w$ are status-consistent if this condition holds. We can now have the following result, which says that if all three-node sets are status-consistent, then the whole graph obeys a form of status-consistency: there is a total ordering of the nodes in which positive edges all go left-to-right, and negative edges all go right-to-left.

THEOREM 2. Let $G$ be a signed, directed tournament, and suppose that all sets of three nodes in $G$ are status-consistent. Then it possible to order the nodes of $G$ as $v_{1}, v_{2}, \ldots, v_{n}$ in such a way that each positive edge $\left(v_{i}, v_{j}\right)$ satisfies $i<j$, and each negative edge $\left(v_{i}, v_{j}\right)$ satisfies $i>j$.

Proof. Following an idea from the previous section, we first reverse the direction of each negative edge in $G$ and give it a positive sign. Notice that if all sets of three nodes were status-consistent before this conversion, they remain status-consistent after this conversion.

Let $G^{\prime}$ be the resulting graph; note that $G^{\prime}$ has only positive edges. If any three-node subgraph in $G^{\prime}$ were to form a directed cycle, then the three nodes in this cycle would violate status-consistency. Thus, all three-node subgraphs of $G^{\prime}$ are acyclic. Applying a wellknown theorem on tournaments (see e.g. the opening exposition in [5]) it follows that $G^{\prime}$ itself is acyclic. Thus, we can find a topological ordering $v_{1}, v_{2}, \ldots, v_{n}$ of $G^{\prime}$.

Finally, we claim that this ordering satisfies the conditions of the theorem. Indeed, if an edge $\left(v_{i}, v_{j}\right)$ is positive in $G$, then it is also an edge in $G^{\prime}$, so by the property of the topological ordering we have $i<j$. And if an edge $\left(v_{i}, v_{j}\right)$ is negative in $G$, then $\left(v_{j}, v_{i}\right)$ is an edge of $G^{\prime}$, whence $i>j$ as required. .

Searching for Evidence of Global Balance and Status. Both Theorem 1 and 2 have more elaborate generalizations, when the underlying graph is not completely connected, by generalizing the respective three-node conditions to arbitrary cycles. Under these generalized conditions the conclusions remain the same: when balance holds, we should expect to see a network that is divided into two mutually opposed factions of friends, and when status holds, we should expect to see a network whose edges respect a global ordering. We therefore take these two basic patterns - a division into two factions, and a global ordering — as potential "signatures" for the effects of balance and status respectively at a global level. Of course, at best we expect to see balance and status holding in an approximate sense, and so we search for approximate versions of these two global signatures, rather than exact versions.

For balance theory, we attempt to partition the graph into two sets to maximize the following objective function: the number of positive edges with both ends in the same set, plus the number of negative edges with ends in opposite sets. (We will also say that those edges that contribute to the objective function are satisfied by the partition.) We develop the following maximization heuristic 


\begin{tabular}{l||r|r|r} 
Balance & Epinions & Slashdot & Wikipedia \\
\hline \hline Netwok & 0.8344 & 0.8105 & 0.7809 \\
Permuted & 0.8562 & 0.7779 & 0.7866 \\
Rewired & 0.8993 & 0.8310 & 0.8316 \\
\hline Status & Epinions & Slashdot & Wikipedia \\
\hline \hline Network & 0.7905 & 0.8221 & 0.8538 \\
Permuted & 0.7241 & 0.7568 & 0.7767 \\
Rewired & 0.6377 & 0.6644 & 0.6321
\end{tabular}

Table 8: Fraction of edges satisfying global balance and status.

for this problem. We start by randomly partitioning the nodes into two sets. Then we repeatedly pick a random node, and change the set it belongs to if that would increase the value of the objective function. We run this procedure many times from different initial starting sets. On our datasets, we found experimentally that the variance of the solution is very small, as the heuristic quickly converges to a solution whose objective function value is within a few hundred of the best solution found over all runs. Note there is a trivial solution that would simply declare one of the sets to be the empty set and the other to be the full node set; this would achieve an objective function value equal to about $80 \%$ of the edges, since in our datasets about $80 \%$ of the edges are positive, and the positive edges would be precisely those that are satisfied by this partition.

For status theory we employ a different heuristic. First, as in the proof of Theorem 2 we replace each negative edge with a positive edge pointing in the opposite direction, thus obtaining a directed network with only positive edges. In this transformed graph, the goal is to find an ordering of the nodes that maximizes the number of edges pointing from a node earlier in the ordering to one that is later in the ordering. (Again, we will refer to such edges as being satisfied by the ordering.) This is precisely the Maximum Acyclic Subgraph Problem, which is known to have strong inapproximability bounds: a random ordering achieves an objective function value equal to half the total number of edges in expectation, and it is computationally hard to do asymptotically better than this in the worst case [9]. Of course, our datasets are not necessarily worst-case instances - indeed, status theory suggests they may have additional structure - and we employ the following heuristic. We start with a random ordering; we then repeatedly pick a random pair of nodes and swap their positions in the ordering if that would increase the value of the objective function. Again, we run this heuristic multiple times and take the best solution found.

Evaluating Global Balance and Status. We now use these two heuristic optimization methods to assess the extent to which each of the three networks exhibits global balance and status properties. That is, we ask whether the quality of the partition or ordering we find is better than would be expected from simple baselines derived from our datasets. If the quality is significantly above such baselines, it provides evidence for these structures. We use two such baselines. The first a permuted-signs baseline in which we keep the structure of the network fixed, but we randomly shuffle all the edge signs. The second is a rewired-edges baseline in which we generate a random network where each node maintains the same number of incoming and outgoing positive and negative edges.

Table 8 shows the value of the objective function (as a fraction of the total number of edges) for each of balance and status, and across each of our real networks in comparison to the permuted-signs and rewired-edges baselines. For balance theory, notice that we find objective function values that are comparable to the total fraction of positive edges, which we noted is trivially achievable (and also trivially achievable in the two baselines, which have the same fractions of positive edges). Moreover, if we randomize the network structure while preserving the signed in- and out-degrees, we obtain a network that actually achieves a greater objective function value under our heuristics. Taken together, this suggests that there is very little evidence for the global presence of structural balance in our three network datasets.

For the global version of status theory, shown in the second part of Table 8 we see quite a different picture. Roughly, we are able to find orderings for all three datasets that satisfy about $80-85 \%$ of all edges. This is much higher than the $50 \%$ obtainable from a random ordering; and moreover, it is significantly better than the performance on either of our two baselines. Thus, we do find evidence for an approximate global status ordering in the real datasets, compared to baselines derived from random variations of them.

Overall, then, there is evidence for link formation consistent with a global status ordering on the nodes, in which positive links tend to point left-to-right in this ordering, while negative tend to point right-to-left in this ordering. On the other hand, we can find no significant evidence for the kind of partitioning into factions that balance theory suggests at a global level. This forms an intriguing contrast with our results at a local level, where there was evidence for both balance and status. There is no contradiction here, since the fidelity of balance and status at a local level is only approximate, but it does raise interesting questions that suggest the need for more powerful and general ways to relate the local structure of sign patterns to more global forms of structure.

\section{PREDICTING POSITIVE EDGES}

In the introduction we noted that the sign prediction problem considered in this paper is closely related to the link prediction problem of inferring latent relationships that are present but not recorded by explicit links [16]. We now turn to this problem in order to investigate the role of negative edges here as well. In particular we consider the question of whether information about negative links can be helpful in predicting the presence or absence of an unobserved positive link. In other words, how useful is it to know who a person's enemies are, if we want to predict the presence of additional friends?

Specifically, suppose we are given a social network where the task is to predict the presence or absence of a positive edge between two individuals. This is analogous to the experiments above, only now it is the presence or absence of an edge in some context rather than the sign of an edge that is to be predicted. We consider two cases. In the first case, only information about the positive edges is used to predict the presence or absence of a hidden positive edge, whereas in the second case information about both the positive and negative edges is used for the prediction.

We use the machine learning framework developed in previous sections to build classifiers that predict whether there exists a positive edge between a pair of nodes. We train two sets of models using the same features (16Triads) but in one case we use the full network with positive and negative edges, while in the other case we use only the positive edges. We then devise the following experiment. For a positive edge $(a, b)$ we pick a corresponding pair of nodes $(c, d)$ that are not connected by an edge but have the same number of common neighbors (embeddedness) as $(a, b)$. Then we formulate a binary classification problem where we are given a pair of nodes and the goal is to determine whether the positive edge is present; that is, we aim to distinguish between pairs of nodes $(a, b)$ that are connected by a positive edge and pairs of nodes $(c, d)$ that are not connected by an edge.

For each pair of nodes we compute two sets of features. For the first set of features we include the information from positive and negative edges by computing the features counting the frequency of each of 16 distinct signed directed triads between a pair of nodes. For the second set of features that are based on only positive edges we simply compute the frequencies of 4 directed paths composed of only positive edges (namely, FFpp, FBpp, BFpp, BBpp). 


\begin{tabular}{l||r|r|r} 
Features & Epinions & Slashdot & Wikipedia \\
\hline \hline Positive edges & 0.5612 & 0.5579 & 0.6983 \\
Positive and negative edges & 0.5911 & 0.5953 & 0.7114
\end{tabular}

Table 9: Predicting the presence of a positive edge.

We then train and evaluate two models: one trained only on features based on positive edges, and the other trained on the whole set of features that also include evidence coming from the negative edges. Our goal here is to understand how information about negative relationships affects the overall performance of predicting existence of positive edges.

Table 9 shows the predictive accuracy for the above task. Since we pick an equal number of edges and non-edges, random guessing has an accuracy of 0.50 . A logistic regression model using only the features based on the positive edges improves the performance to about 0.56 for Epinions and Slashdot, while it gives a much higher boost in Wikipedia, where the classification accuracy almost 0.70 . This is somewhat surprising as we previously saw that for sign prediction Wikipedia was more difficult than the other datasets.

Next we consider a logistic regression model that is trained on features based on both positive and negative edges. This model scores 0.59 on Epinions and Slashdot, while it improves the performance on Wikipedia to 0.71 . This means that if we use information about negative edges for predicting the presence of positive edges in Epinions and Slashdot we get 3 percentage point improvement in absolute terms, and a 50\% increase in the boost relative to random guessing, compared to a model based only on positive edges.

These results clearly demonstrate that in some settings there is a significant improvement to be gained by using information about negative edges, even to predict the presence or absence of positive edges. Thus it is often important to view positive and negative links in an on-line system as inter-related, rather than as distinct noninteracting features of the system.

\section{CONCLUSION}

We have investigated some of the underlying mechanisms that determine the signs of links in large social networks where interactions can be both positive and negative. By casting this as a problem of sign prediction, we have identified principles that generalize across multiple domains, and which connect to social-psychology theories of balance and status. Moreover, our methods for sign prediction yield performance that significantly improves on previous approaches. At a global level, we have seen that there is evidence in all of our datasets for an approximate global status ordering on nodes, while we find in contrast that there is essentially no evidence for a global organization of these networks into opposing factions; this suggests that balance is operating more strongly at a local level than at a global one. Finally, we have seen that employing information about negative relationships can be useful even for tasks that involve only the positive relationships in the network, such as the problem of link prediction for positive edges.

There are a number of further directions suggested by this work. A first one is of course to explore methods that might yield still better performance for the basic sign prediction problem, and to understand whether the features that are relevant to more accurate methods help in the further development of social theories of signed links. We are also interested in strengthening the connections between local structure and global structure for signed links. Finally, as noted at the outset, the role of positive and negative relationships in on-line settings is not limited to domains where they are explicitly tagged as such.

Acknowledgements. We thank Michael Macy for valuable discussions, and the anonymous reviewers for a number of comments and suggestions that helped to improve the paper. Research was sup- ported in part by the NSF grant IIS-0705774, IBM Faculty Award, gift from Microsoft Research and Yahoo! Research Alliance grant.

\section{REFERENCES}

[1] T. Antal, P. Krapivsky, and S. Redner. Social balance on networks. Physica D, 224(130), 2006.

[2] M. J. Brzozowski, T. Hogg, and G. Szabó. Friends and foes: ideological social networking. In Proc. 26th CHI, 2008.

[3] M. Burke and R. Kraut. Mopping up: Modeling wikipedia promotion decisions. In Proc. CSCW, 2008.

[4] D. Cartwright and F. Harary. Structure balance: A generalization of Heider's theory. Psych. Rev., 63, 1956.

[5] M. Chudnovsky, P. Seymour, and B. D. Sullivan. Cycles in dense digraphs. Combinatorica, 28(1):1-18, 2008.

[6] D. Cosley, D. Frankowski, S. B. Kiesler, L. G. Terveen, and J. Riedl. How oversight improves member-maintained communities. In Proc. 23rd CHI, pages 11-20, 2005.

[7] J. A. Davis. Structural balance, mechanical solidarity, and interpersonal relations. Am. J. Soc., 68:444-462, 1963.

[8] R. V. Guha, R. Kumar, P. Raghavan, and A. Tomkins. Propagation of trust and distrust. In Proc. 13th WWW, 2004.

[9] V. Guruswami, R. Manokaran, and P. Raghavendra. Beating the random ordering is hard: Inapproximability of maximum acyclic subgraph. In Proc. 49th IEEE FOCS, 2008.

[10] Z. Gyöngyi, H. Garcia-Molina, and J. Pedersen. Combating web spam with trustrank. In $V L D B$ '04, 2004.

[11] F. Heider. Attitudes and cognitive organization. J. Psych., 21:107-112, 1946.

[12] S. D. Kamvar, M. T. Schlosser, and H. G. Molina. The eigentrust algorithm for reputation management in $\mathrm{p} 2 \mathrm{p}$ networks. In Proc. 12th WWW, pages 640-651. ACM, 2003.

[13] J. Kunegis, A. Lommatzsch, and C. Bauckhage. The Slashdot Zoo: Mining a social network with negative edges. In Proc. 18th WWW, pages 741-750, 2009.

[14] C. Lampe, E. Johnston, and P. Resnick. Follow the reader: filtering comments on slashdot. In Proc. 25th CHI, 2007.

[15] J. Leskovec, D. Huttenlocher, and J. Kleinberg. Signed networks in social media. In Proc. 28th CHI, 2010.

[16] D. Liben-Nowell and J. Kleinberg. The link-prediction problem for social networks. J. Amer. Soc. Inf. Sci. and Tech., 58(7):1019-1031, 2007.

[17] S. Marvel, S. Strogatz, and J. Kleinberg. Energy landscape of social balance. Physical Review Letters, 103, 2009.

[18] P. Massa and P. Avesani. Controversial users demand local trust metrics: an experimental study on epinions.com community. In $A A A I$ '05, pages 121-126. AAAI Press, 2005.

[19] M. E. J. Newman. The structure and function of complex networks. SIAM Review, 45:167-256, 2003.

[20] B. Pang and L. Lee. Opinion Mining and Sentinment Analysis. Number 2(1-2) in Foundations and Trends in Information Retrieval. Now Publishers, 2008.

[21] P. Resnick and H. R. Varian. Recommender systems. Comm. ACM, 40(3):56-58, 1997.

[22] M. Richardson, R. Agrawal, and P. Domingos. Trust management for the semantic web. In ISWC, 2003.

[23] S. Wasserman and K. Faust. Social Network Analysis: Methods and Applications. Cambridge Univ. Pr., 1994.

[24] F. Wu and B. A. Huberman. How public opinion forms. In Proc. 4th WINE, pages 334-341, 2008.

[25] L. Xiong and L. Liu. Peertrust: supporting reputation-based trust for peer-to-peer electronic communities. IEEE Trans. Knowl. Data Engr., 16(7):843-857, 2004. 